\title{
LA PRECIPITACIÓN INTENSA VISTA DESDE LA CRITICALIDAD AUTO-ORGANIZANDA Y LAS TRANSICIONES DE FASE CONTINUAS: UN NUEVO ENFOQUE DE ESTUDIO
}

\author{
INTENSE PRECIPITATION SINCE AUTO-ORGANIZED CRITICALITY AND \\ CONTINUOUS PHASE TRANSITIONS: A NEW APPROACH
}

\author{
Sheila Serrano ${ }^{1,2}$ y Leonardo Basile ${ }^{1}$
}

\author{
${ }^{1}$ Maestría en Física, Departamento de Física, Escuela Politécnica Nacional, Ladrón de Guevara E11-253, Telf: (593-2) 2507144, \\ Quito, Ecuador. \\ ${ }^{2}$ Centro de Investigación en Modelamiento Ambiental, Universidad Politécnica Salesiana, Av. 12 de Octubre N24-22 y Wilson, \\ Telf. (593-2) 3962800, Quito, Ecuador. \\ Autor para correspondencia: leonardo.basile@epn.edu.ec
}

Manuscrito recibido el 18 de abril de 2012. Aceptado, tras revisión, el 17 de junio de 2012.

\section{Resumen}

La criticalidad auto-organizada, en inglés Self-Organized Criticality (SOC) es un modelo teórico reciente que describe a los sistemas complejo y ocurre generalmente cerca de las transiciones de fase continuas. Según esta teoría, un parámetro de orden se incrementa siguiendo una ley de potencia cuando un parámetro de sintonización pasa por un valor crítico; al acoplarse estos parámetros, el punto crítico se convierte en un atractor y resulta una SOC. Asimismo, la escala del parámetro de orden diverge. Aunque este concepto ha sido aplicado a campos muy diversos, que van desde la geofísica hasta la economía, los fenómenos climáticos aún se siguen considerando como sistemas caóticos cuyo comportamiento, marcado por el cuasi-equilibrio de Arakawa y Schubert (1974), está lejos de la auto-organización. Bajo este contexto, la presente revisión busca dar un formulismo efectivo a los fenómenos que involucran las precipitaciones intensas, utilizando la teoría de la SOC y las transiciones de fase. Así, se argumenta que al llegar a un valor crítico de vapor de agua (el parámetro de sintonización) se genera una transición de fase continua fuera del equilibrio hacia un régimen de convección y precipitación intensas (el parámetro de orden), con regiones de correlación con magnitudes de decenas a cientos de kilómetros (Peters y Neelin, 2006b). Así se logra una relación de potencia entre la precipitación intensa y los datos el vapor de agua.

Palabras claves: Criticalidad auto-organizada, transiciones de fase continuas, precipitación intensa.

\begin{abstract}
The self-organized criticality (SOC) is a recent proposed theoretical model for complex systems. Critical phenomena occur near phase transitions. According to this theory, when an ordered parameter increases as a power law, a tuning parameter crosses a critical value. When these parameters couple, the critical point becomes an attractor and the result is a SOC phenomenon. As a characteristic, their scale diverge. Although this concept has been applied in many different processes, ranging from geophysics to economy; in meteorology the physical process is still being considered as chaotic systems. In this case the behavior, marked by the quasi-equilibrium (Arakawa y Schubert, 1974) is far from being self-organized, with uncertainties that grow exponentially. In this context, the present review aims to propose an effective formalism to intense precipitation phenomena using SOC and continuous phase transitions theory. We suggested that the achievement of a critical value of water vapor (parameter tuning) generates a continuous phase transition that yields into a regime of strong convection and precipitation (the order parameter), with correlated regions of hundreds of kilometers (Peters y Neelin, 2006b) that follow a power law relation between intense precipitation and water vapor data.
\end{abstract}

Keywords: Self-organized criticality, continuous phase transitions, intense precipitation.

Forma sugerida de citar: Serrano, S. y L. Basile 2012. La precipitación intensa vista desde la criticalidad autoorganizanda y las transiciones de fase continuas: un nuevo enfoque de estudio. La Granja. Vol. 15(1): 5-18. ISSN: 1390-3799. 


\section{Introducción}

La criticalidad autoorganizada (SOC por sus siglas en inglés, Self Organized Criticality), describe como grandes sistemas dinámicos tienden a un estado crítico. Estos sistemas, llamados complejos contienen muchas partes, relativamente independientes, altamente interconectadas e interactivas; de las cuales se requiere un gran número para generar sistemas autoorganizados, replicantes, adaptativos con memoria y capacidad de aprendizaje. Estos sistemas, gracias al proceso de autoorganización se hacen más ordenados y con más información que otros sistemas -caóticos fuera o cercanos al equilibrio termodinámico (Cowan et al., 1994). Así, la SOC es una de las teorías de la complejidad, ciencia que engloba la teoría de los sistemas adaptativos complejos, la dinámica no lineal, la teoría de los sistemas dinámicos, la teoría del no-equilibrio y el caos. La SOC fue propuesta por Bak, Tang y Wiesenfeld en 1987, trabajo que se ha convertido en uno de los más citados de todos los tiempos (Reynoso, 2007).

Por otro lado, el comportamiento del tiempo y del clima se ha caracterizado por su naturaleza caótica e impredecible, ya que al ser la atmósfera un fluido, su dinámica responde a una serie de ecuaciones no lineales que no tienen una solución analítica (Steward, 1997). Sin embargo, existe evidencia científica de que para el caso de las precipitaciones lluviosas, estos eventos se aproximen más a sistema crítico que a uno caótico y que por lo tanto exhiban un nivel de organización e información superior (Peters y Neelin, 2006b).

De esta manera, la presente investigación realizará una revisión general al concepto de criticalidad autoorganizada, y otros conceptos de los sistemas complejos y la física estadística, planteándolos como una alternativa de análisis a los fenómenos climáticos en general, y de manera particular para el caso de la lluvia intensa, fenómenos regularmente estudiados sólo desde la dinámica de fluidos.

Los conceptos fundamentales se expondrán en la sección 2, abarcando el experimento canónico de la pila de arena, los conceptos de criticalidad autoorganizada, leyes de potencia, ruido $1 / f$, y los exponentes que aparecen en estas distribuciones "sin escala", además de las nociones de transiciones de fase. En la sección 3 se verá cómo aplicar los conceptos de criticalidad y transiciones de fase al estudio de la precipitación lluviosa, mientras que en las secciones
4 y 5 se los relacionará a las nociones de complejidad, adaptabilidad, entropía y negentropía.

\section{Conceptos fundamentales}

\subsection{El experimento de la pila de arena}

El ejemplo canónico de criticalidad es la pila de arena, como lo explica Bak (1996) en su libro titulado How nature works: the science of self-organized criticality (Ver Figura 1).

El experimento consiste en un sistema construido por la lenta adición de partículas de arena sobre un pequeño montón (parte a de la Figura 1). Al ir añadiendo arena la pendiente de la pila se incrementa hacia un valor crítico y en este punto se generan avalanchas de todos los tamaños. Al principio, cuando la pila era plana, era factible realizar una descripción aislada de cada avalancha, sin embargo, en el estado crítico, la descripción de las avalanchas debe considerarse holísticamente, ya que interacciones vinculan a partes más alejadas del sistema, y en este caso sólo una descripción de la pila de arena como un todo se puede aplicar. Este paradigma ha sido estudiado por varios autores con simulaciones numéricas computacionales a través del modelo del los "automatones de la pila de arena".

Este modelo es bastante simple, e inicia definiendo una grilla bidimensional sobre unos límites específicos. A continuación, se define una variable entera $Z$ que representa la altura o la pendiente de la pila. Si se incrementa $Z$ en algún punto (por la adición de arena) y no se ha superado un valor crítico, se puede seguir aumentando $Z$. Si se ha logrado el valor crítico, hay que reducir $Z$ en cuatro unidades y enviar una unidad a los cuatro vecinos más cercanos. Por otro lado, si $Z$ excede el valor crítico en alguno de los sitios vecinos es necesario repetir el experimento hasta que la "avalancha" se detenga para que en ningún lugar se supere el valor crítico de $Z$. Después, se continúa con la adición de arena para generar más avalanchas (Bak y Sneppen, 1993).

Cuando las avalanchas se dan en los límites de la grilla, existirán unidades de $Z$ que saldrán del sistema. Éstas serán las avalanchas de las cuales se registrará su magnitud. Al registrar la magnitud de las avalanchas en un intervalo de tiempo, se obtendrá una gráfica como la que se muestra en la Figura 1.b. Esta errática gráfica es denominada ruido uno sobre 
$f(1 / f)$, y es muy diferente al ruido blanco, ya que se encuentran descritas magnitudes de todos los tamaños.

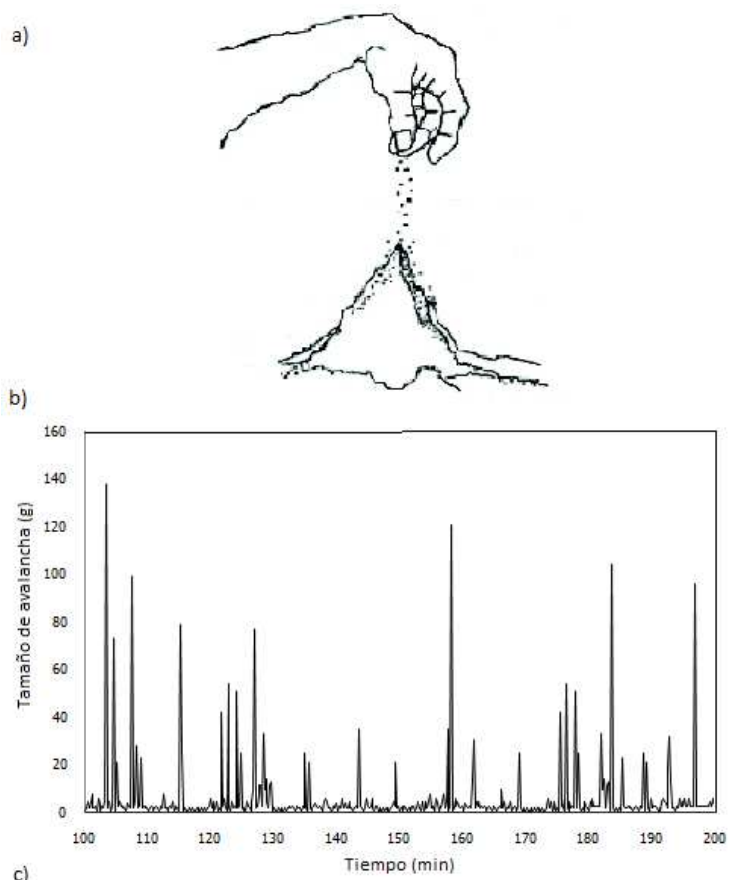

c)

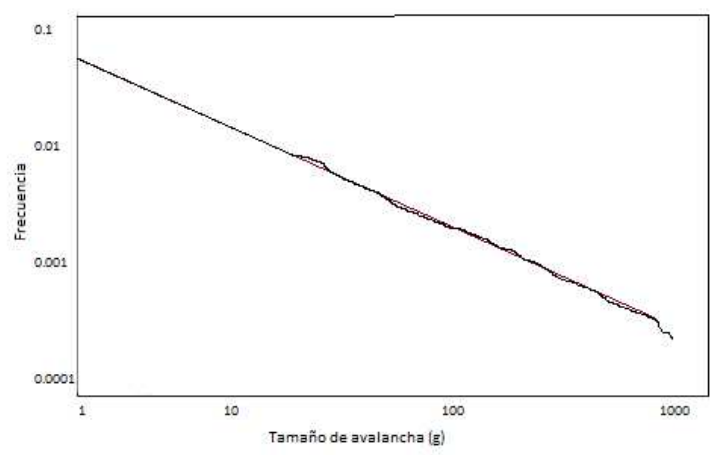

Figura 1. a) Modelo de la pila de arena desarrollado por Bak, Chen y Wiesenfeld; b) serie de tiempo que muestra la magnitud o tamaño de las avalanchas generadas en el modelo; c) distribución de las Magnitudes en una escala doble logarítmica, en la que se aprecia la ley de potencia. Adaptado de Bak (1996).

Se lo puede representar como una señal compuesta de todas las frecuencias descrita por la función

$$
1 / f^{\phi}
$$

donde el exponente está en el rango de $0,6<\phi<2$.

\subsection{Distribuciones "sin escala" y leyes de potencia}

Si se quisiera determinar el tamaño de una avalancha típica, se podría generar un histograma que cuente el número de avalanchas en función de su tamaño y se obtendría una gráfica como la que se presenta en la Figura 1.c. La gráfica está en escala doble logarítmica e indica que los tamaños más pequeños de las avalanchas son los más frecuentes y que las avalanchas más grandes o catastróficas son las menos frecuentes. Se dice entonces que este tipo de eventos siguen una "ley de potencias", ya que están determinadas por una ecuación del tipo

$$
N(E)=E^{b}
$$

donde $E$ es el tamaño de la avalancha, $N(E)$ la frecuencia, y $b$ es un exponente negativo.

En general, comportamientos con las características de la pila de arena aparecen por doquier en la naturaleza, Raup (1986) ha mostrado que la evolución biológica es intermitente con largos períodos estáticos interrumpidos abruptamente por eventos de extinción. Hay muchos eventos pequeños y muy pocos eventos de gran magnitud: como la extinción de los dinosaurios hace 50 millones de años, la gran extinción Pérmica hace 200 millones de años, y la explosión Cámbrica hace 550 millones de años. La distribución de estos eventos también es similar a la ley de Gutenberg-Richer (Olami et al., 1992) que determina la energía liberada en los terremotos. Asimismo, Mandelbrot (1982) observó que la distribución de las fluctuaciones de los precios del algodón y las acciones ferroviarias no es gausiana sino que obedece una ley de potencias. Se hace evidente entonces, que el mecanismo que genera eventos de grandes magnitudes o "catastróficos" es el mismo que genera eventos de menor magnitud, y que no es necesario ningún cataclismo externo para producirlos $(\mathrm{Pu}-$ yeyo, 2011; Bak y Sneppen, 1993; Adami, 1955; Kron y Grund, 2009; Sekar, 2007).

Una distribución en ley de potencia también es conocida como distribución "sin escala", debido a que la ley de potencia es la única distribución que es la misma en cualquiera que sea la escala donde se la analice (Newman, 2005).

Para entender este concepto, supongamos que una distribución de frecuencias $f(x)$, de una cantidad $x$, satisface una condición de la forma:

$$
f(c x)=g(c) f(x)
$$


para cualquier valor $c$. Así, si se incrementa la escala de los valores de $x$ por una constante $c$, el comportamiento de la distribución $f(x)$, permanecerá inalterado, excepto por una constante multiplicativa general.

Por ejemplo, podemos decir que una avalancha de $20 \mathrm{~g}$ es 10 veces menos frecuente que una avalancha de $2 \mathrm{~g}$. Si aumentamos la escala de la magnitud de la avalancha en un factor de 10, la proporción se mantendrá: es decir, que una avalancha de $200 \mathrm{~g}$ será 10 veces menos frecuente que una de $20 \mathrm{~g}$. Y una de $2000 \mathrm{~g}$ será 10 veces menos frecuente que una de $200 \mathrm{~g}$ y así sucesivamente. Es decir que la distribución de las frecuencias no depende de la escala con la que sea tomada la magnitud del evento.

Eventos de la naturaleza que no poseen una escala determinada, son considerados fractales; y sus propiedades reproducen la misma estructura del universo, los paisajes montañosos, la biología, las nubes y las cuencas hidrográficas, entre muchas otras estructuras. Y aunque se han hecho esfuerzos por caracterizarlos geométricamente, aún no es claro su origen dinámico (Turcotte, 1985).

\subsection{La criticalidad autoorganizada}

Todos los sistemas de la naturaleza descritos anteriormente son grandes, dinámicos y con muchas partes que se encuentran en interacción. Son también disipativos, en donde existe un suministro constante de energía de una fuente externa: el movimiento de las placas tectónicas en el caso de los terremotos, el empuje en el caso de la turbulencia, alimento y energía en biología, etc. Esta energía es eventualmente usada o disipada en algún punto del sistema.

Desde la teoría, el punto exacto en el que un sistema no manifiesta una escala típica (la escala diverge, sistema sin escala), es conocido como punto crítico de una transición de fase. Más específicamente de una transición de fase continua (ya que también existen otros tipos de transiciones, como se verá en el apartado 2.4). Todo lo que ocurre en la vecindad de una transición de fase continua es conocido como fenómeno crítico, del cual, las distribuciones en ley de potencia son un ejemplo (Newman, 2005).

Lo común es que este punto crítico esté determinado por algún parámetro móvil de sintonización -como la temperatura para el caso del ferromagnetismo- que al alcanzar un valor determinado, genera la criticalidad. Pero como el mismo Bak (1994) menciona, "en los fenómenos naturales, no hay quien se encargue de sintonizar estos parámetros, entonces cómo se origina la criticalidad?"

Para entender estos comportamientos, en 1991, Bak y Chen propusieron que los sistemas dinámicos alimentados lentamente, con muchos grados de libertad, se organizan naturalmente hacia un estado crítico, caracterizado por tener avalanchas de todos los tamaños y obedeciendo una frecuencia en ley de potencia. El estado crítico es un atractor, ya que el sistema tiende irremediablemente a él sin importar las condiciones iniciales: en el caso de la pila de arena, si la pendiente de la pila es pequeña estado subcrítico-, irá acumulando más arena; si es muy grande -estado supercrítico- se generarán avalanchas. Tanto los apilamientos subcríticos como los supercríticos se ven pues, espontáneamente arrastrados hacia el estado crítico. Así, a diferencia de la física en equilibrio, en donde el estado crítico es un caso excepcional, en la física del no equilibrio, la criticalidad es el estado típico de la materia.

Sin embargo cabe la pregunta: ¿se mantiene la criticalidad si se cambia algún parámetro en el sistema? La respuesta es sí, no importa si se cambia la arena seca por húmeda o si se construyen barreras para evitar las avalanchas; si se alimenta aleatoriamente a la pila o de una manera determinada sobre un solo punto, el sistema siempre evolucionará hacia su propio estado crítico. Se presenta aparentemente una paradoja: el sistema es inestable en muchas de sus distintas localidades, pero el estado crítico es absolutamente robusto (Ibañez, 2007). Por una parte, ciertos rasgos locales cambian continuamente a causa de las avalanchas. Por otra, algunas propiedades estadísticas, como la distribución por tamaños de las avalanchas, no se alteran en el tiempo (Lucas, 1999).

\subsection{Significado de los exponentes en las leyes de potencia y el ruido $1 / f$}

Claramente los exponentes que aparecen en las leyes de potencia y en el ruido $1 / f$ deben tener un significado físico interesante, mismo que se hace evidente al abordar el modelo de Olami, Christensen y Feder, propuesto en 1992; basado en el modelo canónico de la pila de arena pero aplicado al caso de los terremotos. 
Para simular la corteza terrestre, este modelo considera bloques bidimensionales conectados por resortes -en el que el tamaño del bloque no representa una escala de longitud intrínseca del sistema-. Sobre cada bloque $(i, j)$ actúa una fuerza $F_{i, j}$ que se incrementa uniformemente por un monto infinitesimal por unidad de tiempo, simulando así una acumulación de energía por el movimiento de las placas tectónicas. Eventualmente, la fuerza en un sitio $(i, j)$ excederá un valor crítico $F c$ de ruptura. Esta ruptura se simulará repartiendo toda la fuerza que se encontraba en el sitio $(i, j)$ sobre cada uno de sus cuatro vecinos $(i, j \pm 1)$ y $(i \pm 1, j)$, de la siguiente manera:

$$
F_{i, j} \geq F c \Longrightarrow\left\{\begin{array}{l}
F_{i, j} \rightarrow 0 \\
F_{n n} \rightarrow F_{n n}+\alpha F_{i j}
\end{array}\right.
$$

donde $n n$ denota el conjunto de sitios de los vecinos de $(i, j)$.

Estas ecuaciones, completamente deterministas, representan la transferencia de la fuerza hacia los bloques vecinos, que causará que se vuelvan inestables y se dé a lugar una reacción en cadena. Esta reacción en cadena es el terremoto. Cuando el terremoto se detiene el sistema se mantiene quieto hasta que la fuerza en otro punto exceda el valor crítico, y un nuevo evento inicie, una y otra vez.

En este caso, el valor de $\alpha$ está directamente relacionado con los parámetros de elasticidad de la corteza terrestre. Para el valor de $\alpha=1 / 4$, se puede decir que la fuerza se conserva i.e., el monto de fuerza en el sitio $(i, j)$, iguala al monto total de fuerza que ganan los cuatro sitios vecinos. Sin embargo, se ha encontrado que la criticalidad prevalece para valores de $\alpha$ menores que 0.05 , es decir donde sólo existe una conservación del $20 \%$ (Bak, 1994), hecho que se espera de sistemas disipativos.

Por otro lado, el exponente b de la Ecuación 2, correspondiente a la pendiente de la ley de potencias que se muestra en la Figura 1c. está relacionado con $\alpha$, de la forma: $b=1-\alpha$. Es decir que la pendiente $\mathrm{b}$ depende del grado de disipación de la energía en el sistema, y por lo tanto no existirá criticalidad para el caso de sistemas conservativos.

Abordando el segundo punto de discusión, el ruido $1 / f$, se ha mencionado que éste puede ser pensado como la superposición de avalanchas que ocurren en un estado de SOC. Para ilustrar este hecho, Chistensen et al., analizan en 1991 el tiempo de vida de una avalancha, con pesos:

$$
\Lambda(t)=\Sigma s^{2} P(s=s, T=t)
$$

donde $P(S, T)$ es la probabilidad de que una avalancha de tamaño $S$ tenga una duración de $T$ ( $T$ en unidades de tiempo). Se ha mostrado que si $\Lambda$ tiene un comportamiento en potencia $\Lambda(t)=t^{u}$, entonces la potencia espectral será:

$$
S(f)=f^{-(u+1)}, \quad \text { para }-1<u<1 .
$$

Así el exponente de $f$, phi estará determinado por la duración de los fenómenos de disipación de energía (avalanchas), según la relación: $\phi=-(u-1)$.

En la naturaleza se han reportado exponentes de $1 / f$ con valores que están en el intervalo 0.6-2.0. Sin embargo, hay que recalcar que estos exponentes dependen de los parámetros del modelo, y que por lo tanto no son universales. Estos hechos muestran que los eventos críticos carecen también de escala temporal.

\subsection{Transiciones de fase}

En general, transición de fase es la transformación de un estado de la materia con propiedades físicas homogéneas (fase) a otro con propiedades diferentes. Antiguamente, se pensaba que los estados de la materia eran tres: sólido, líquido y gaseoso (después se agrega el estado de plasma), sin embargo, el número de estados de la materia que la ciencia actual ha descubierto se ha multiplicado enormemente, hablando estrictamente: los magnetos son un diferente estado de la materia, así como los metales son diferentes de los aislantes. Los superconductores y los superfluidos son interesantes nuevos estados, así como existe una familia completa de diferentes estados de la materia correspondientes a los cristales líquidos -como los que se usan en los relojes digitales-, en donde se han identificado 200 tipos diferentes, sin mencionar a los quasicristales. Hay estados desordenados de la materia como los spines de vidrios y los estados fraccionarios del efecto Hall cuántico, como los quarks, y otros estados desarrollados desde la física de partículas. En fin, actualmente se cuentan 500 estados de la materia (Sethna, 2010).

Pero, qué es lo que distingue un estado de la materia de otro? En realidad, se ha determinado que si 
dos materiales tienen diferente simetría, se los puede considerar en diferentes fases. Por ejemplo, si se analiza un cubo y una esfera, claramente se ve que la esfera es más simétrica que el cubo, ya que ésta puede ser rotada en cualquier ángulo y permanecer invariante, es decir, que mantiene una invariancia rotacional. Mientras que el cuadrado únicamente puede ser rotado $90^{\circ}, 180^{\circ}$ y $270^{\circ}$ respecto a los tres ejes que pasan a través de sus caras. Se puede girar por $120^{\circ}$ o $240^{\circ}$ respecto a las esquinas y por $180^{\circ} \mathrm{si}$ se rota alrededor del eje que pasa desde el centro a través de cualquiera de los 12 bordes del cubo. Así, el cubo y la esfera poseen diferente simetría, como la pueden presentar dos estados de la materia diferentes.

Cada fase estará determinada por una propiedad física importante la cual está determinada por un parámetro de orden. Al ocurrir una transición de fase, será este parámetro de orden el que definirá cuantitativamente esta transición.

Asimismo, en la coexistencia cada fase tiene sus propiedades termodinámicas bien definidas (como temperatura y la presión constantes), pero estas fases son sistemas abiertos que acumulan y disipan materia y/o energía, por lo que de debe cumplirse que los potenciales químicos de cada fase deben ser iguales. Esto significa que tanto el potencial químico $\mu$ como la energía libre de Gibbs $G$ deben cambiar al ocurrir una transición de fase. Las transiciones se clasifican según la continuidad de las derivadas del potencial de Gibbs: cuando hay cambios de estado discontinuos (primeras derivadas de $G$ discontinuas), tenemos una transición de fase de primer orden y los estados correspondientes a cada fase se hallan en regiones separadas del espacio de configuraciones termodinámicas; cuando los cambios de estado son continuos (derivadas superiores discontinuas), nos encontramos con transiciones de fase continuas o de orden superior, también denominadas fenómenos críticos.

\section{Estudios sobre la precipitación lluviosa}

\subsection{Criticalidad en la precipitación llu- viosa}

Un fluido, como la atmósfera, al cual se le impone una diferencia de velocidad $v$, sobre una escala de longitud $L$, experimenta una transición a un es- tado turbulento. En este estado se generan vórtices con un amplio rango de escalas de longitud. Mientras que la energía se disipa localmente dentro de una pequeña escala de longitud llamada longitud de Kolmogorov, con dimensiones fractales cercanas a cero (Mandelbrot, 1982).

Al igual que los ejemplos abordados anteriormente: pila de arena y terremotos, el fenómeno de la turbulencia en un fluido tiene un comportamiento similar, aunque sus escalas de tiempo y espacio difieren enormemente. En ambos casos la energía entra al sistema de una manera lenta y uniforme y lo abandona localmente, tal como menciona Kagan, en su libro Sismicidad: turbulencia en Sólidos.

De esta manera, se hace evidente la validez de pensar en un proceso SOC, como uno turbulento. Generalmente, la turbulencia en un fluido estaba determinada por las ecuaciones dinámicas no lineales de Navier Stokes, utilizadas ampliamente en la investigaciones de los comportamientos atmosféricos (Palacios et al., 2009). Sin embargo, la naturaleza no analítica de las soluciones de estas ecuaciones hacían ver a los fenómenos atmosféricos como caóticos (Steward, 1997). Generándose así esfuerzos para desarrollar técnicas computacionales que a través de métodos numéricos y una amplia red de monitoreo meteorológico han podido generar avances en la investigación de los comportamientos climáticos, pero sin un modelo teórico que realmente pueda evidenciar la verdadera naturaleza del sistema atmosférico.

Años atrás, en 1974 Arakawa y Schubert, conciben al sistema atmosférico como uno de acumulación de energía generada por los procesos de evaporación del agua, seguido por procesos de disipación de la misma representados por las precipitaciones. De esta manera, y desde un punto de vista estadístico, se evidenciaría un equilibrio entre los procesos de acumulación y disipación de energía -aunque en realidad el sistema está fuera del concepto conocido del equilibrio termodinámico-, por lo que se ha denominado a estos sistemas como de cuasi-equilibrio (QE). Sin embargo, a pesar de que contar con un modelo de esta naturaleza fue valioso, la lluvia seguía siendo considerada parte de un sistema dinámico que tendía irremediablemente al caos y escapaba de las predicciones, sobretodo cuando se trataba de la precipitación intensa (Palacios y Serrano, 2011).

En el año 2002 se hicieron interesantes estudios experimentales para relacionar a la teoría de la SOC

LA GRANJA, Revista de ciencias de la vida, 15(1) 2012: 5-18.

(C) 2012, Universidad Politécnica Salesiana, Ecuador. 
con el fenómeno de la precipitación (Peters y Christensen, 2002; Peters et al., 2002). Se realizó un estudio de la precipitación generada sobre el Mar Báltico con datos de radar Doppler, aplicados sobre un rango de altura que iba de los 50 a los $250 \mathrm{msnm}$ durante todo el año 2001. Cabe indicar que se eligió realizar el trabajo sobre el mar debido a la poca variabilidad térmica del agua.

Los datos de radar utilizados tuvieron una gran precisión que llega incluso a detectar precipitaciones de hasta 10-4 mm (se determinó como precipitación a cualquier masa de agua que caía con velocidad diferente de cero). Detectando de esta manera virgas, es decir, precipitaciones tan pequeñas que se evaporan antes de tocar el suelo.

Asimismo, la resolución temporal también fue alta: $1 \mathrm{~min}$, lo cual permitió detectar la verdadera intensidad de la lluvia, por corta que esta fuera ya que no se ocultaba su verdadera magnitud, que generalmente se perdía al promediarla en periodos de tiempo mayores. Los datos se presentan en la Figura 2.

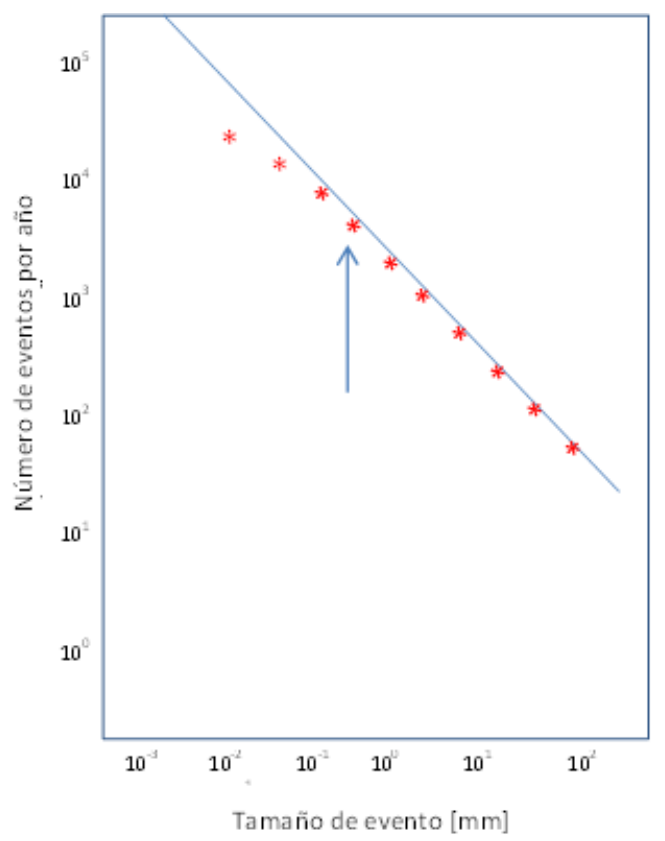

Figura 2. Distribución de las magnitudes de precipitación en una escala doble logarítmica, sobre la cual se aprecia claramente la ley de potencia. La flecha indica el punto de mínima resolución de un pluviómetro normal, bajo el cual, la precipitación puede considerarse como virga. Adaptado de Peters y Christensen (2002) y Peters et al. (2002).
De esta manera Peters et al. (2002) encuentran que la distribución de las magnitudes de las precipitaciones registradas seguían una ley de potencia, tal como ocurría con el modelo de la pila de arena (2). La relación que se encontró fue:

$$
N(M) \propto M^{\tau}
$$

donde $N(M)$ se refiere al número de eventos producidos en el año estudiado, $M$ la magnitud de la precipitación medida en $\mathrm{mm}, \mathrm{y} \tau$ se refiere al exponente de la relación, que en este caso llegó a ser $\tau=1,4$. De esta manera, se infiere que la precipitación es un buen candidato a un sistema en SOC y que por lo tanto dicho marco teórico puede ser considerado en su estudio.

Cabe indicar, que la misma ley de potencia fue encontrada al determinar la distribución de frecuencias de la duración de los eventos lluviosos, encontrándose en este caso, un un exponente de $\tau=1,6$. Asimismo al estudiar el tiempo de duración de los períodos de sequía (días sin precipitación) se obtuvo un exponente $\tau=1,4$. No menos relevantes son los estudios de Bunde et al. (2003) que muestran el comportamiento de la potencia entre las correlaciones de las variaciones de temperatura y sus tiempos de correlación, encontrando exponentes de 0,7.

\section{Transiciones de fase y precipi- tación lluviosa}

La mayoría de tormentas ocurren en escalas de tiempo relativamente cortas y se presentan como eventos extremos con tasas de lluvia que exceden los promedios climatológicos por varios órdenes de magnitud.

Desde la aplicación de la dinámica de fluidos al estudio de la atmósfera, es un hecho conocido que el movimiento de las masas de aire húmedas se da principalmente por convección, y la precipitación algunas veces intensa- que este movimiento ocasiona es sensible a las variaciones del vapor de agua calculadas a lo largo de una columna vertical. Estos valores generalmente son estudiados en grandes escalas de tiempo y espacio; hecho verificado tanto en observaciones reales, como las de Parsons et al. (2000), y en modelos numéricos como en Grabowski (2003). 
En este caso, el efecto del empuje del vapor de agua sobre las nubes, arrastra el aire circundante generando un flujo turbulento. Así, la hipótesis de Peters y Neelin (2006a) plantea que la transición a la convección intensa, acompañada por el inicio de la precipitación intensa, muestra signos de ser una transición de fase continua. Cabe indicar que las transiciones de fase que se dan en un fluido en convección son completamente diferentes a las transiciones de fase discontinuas, típicas de los cambios de estado, como la condensación que se da en las gotas de agua.

De esta manera, el modelo de Peters y Neelin considera a la atmósfera como una grilla tridimensional. Las reglas de conservación de partículas se definen de tal manera de que en cada espacio de la red exista un número definido de partículas, las cuales podrán saltar al espacio vecino si dentro de su propia región se excede un cierto umbral crítico de densidad. Estas simples reglas generarán una transición de fase al llegar a este valor crítico; desde una fase con poca movilidad (en donde eventualmente el sistema llega a una configuración estable) hacia una fase activa (en donde las configuraciones estables ya no son accesibles). El parámetro de sintonización será entonces la densidad de partículas estacionarias, y el parámetro de orden se identifica como la densidad de sitios activos, como indican Marro y Dickman (1999).

Así, la SOC se describe como un atractor de la transición de fase, ya que al estar abiertas las fronteras de la grilla, y asimilar una adición de partículas muy lenta, el parámetro de sintonización se incrementa hacia el valor crítico de densidad, lo cual genera una transición de fase hacia un estado activo que a su vez disipa a las partículas desembocando en una disminución del parámetro de sintonización nuevamente hacia el estado crítico.

El modelo propuesto define al parámetro de orden como a la precipitación, mientras que el parámetro de sintonización queda determinado por la cantidad de vapor de agua. Así, las avalanchas de todos los tamaños (lluvias), serán la característica del estado crítico, que resultan de la proximidad permanente del sistema alrededor de un punto crítico (Pruessner y Peters, 2006).

Esta conjetura coincide tanto con el concepto de transición de fase, como con el de QE (Arakawa y Schubert, 1974). Las pruebas observables de esta continua proximidad a un estado de QE han sido la base de la mayoría de parametrizaciones convectivas en los modelos dinámicos a gran escala y mesoescala, así como mucha de la teoría dinámica tropical desarrollada hace más de dos décadas.

\subsection{Evidencia experimental de las transi- ciones de fase en la precipitación llu- viosa}

Con estos antecedentes, se presenta el trabajo experimental que realizaron Peters y Neeil desde el 2006, tanto desde el punto de vista estadístico (Peters y Neelin, 2006b,a), como del climatológico (Neelin et al., 2009).

Debido a que el valor de vapor de agua, w, depende principalmente de la temperatura, se determina una cantidad de vapor de agua para un promedio de temperaturas característico a una región climática dada dentro de los trópicos, es decir, entre las latitudes $20^{\circ} \mathrm{N}$ a $20^{\circ} \mathrm{S}$, y sobre los océanos, debido a la gran inercia térmica que poseen. En total, se definieron cuatro regiones climáticas correspondientes a los océanos: Pacífico oeste, Pacífico este, Atlántico e Índico.

Los datos de radar fueron recopilados del TRMM (Tropical Rainfall Measuring Mission), que cuentan con una resolución espacial de $0,25^{\circ} \times 0,25^{\circ}$ (lo que equivale a $12,7 \mathrm{~km} \times 12,7 \mathrm{~km}$ ), qque puede considerarse suficientemente alto a nivel espacial. Temporalmente también se cuenta con una buena resolución ya que existen datos cada tres horas. Cabe indicar que estos datos satelitales han sido validados y probados desde estaciones en tierra y en aire por la TSVO (por TRMM Satellite Validation Office, en inglés) con el objetivo de verificar la fiabilidad de los datos (Wolff et al., 2004).

De esta manera, se trabaja con el parámetro conocido como columna de vapor de agua $w$ (que se refiere al volumen agua líquida por área, medido en $\mathrm{mm}$ )determinado como parámetro de sintonización, el promedio de la precipitación $\langle P\rangle(w)$ dada en $\mathrm{mm} / \mathrm{h}$, que está determinado como el parámetro de orden. Estas observaciones se promedian sobre cada grilla y en las tres horas observadas, en cada región durante los cinco años investigados (el promedio se representará por \langle\rangle ).

Así, en la Figura 3 se muestra el comportamiento del promedio de la precipitación $\langle P\rangle(w)$ dada en $\mathrm{mm} / \mathrm{h}$, en función del parámetro de sintonización $w$ 
dado en mm. Aunque el comportamiento para cada región climática es bastante similar, la ley de potencia se hace más evidente al reescalar cada término. Esto se logra multiplicando al parámetro de orden $\langle P\rangle(w))$ por un factor de reescalamiento $f_{P}^{i}, \mathrm{y}$ al parámetro de sintonización $w$ por $f_{w}^{i}$, en este caso el índice $i$ corresponde a cada región climática, tal como se muestra en la Figura 4.

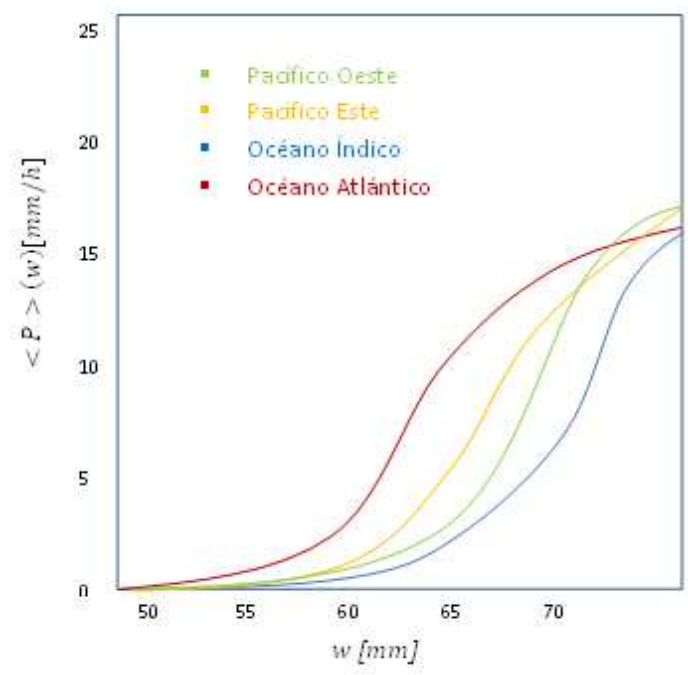

Figura 3. Parámetro de orden (promedio de la precipitación $\langle P\rangle(w)$ ) en función del parámetro de sintoninzación (vapor de agua $w$ ), para cada región climática estudiada en los trópicos. Los datos se presentan sin reescalamiento. Adaptado de Peters y Neelin (2006a).

Después del reescalamiento es evidente que los parámetros de orden y sintonización siguen una ley de potencia del tipo:

$$
\langle P\rangle(w)=a\left(w-w_{c}\right)^{b} .
$$

Donde $a$ y $w_{c}$ son constantes que dependen del sistema climatológico estudiado, y $b$ es un exponente universal. Cabe indicar que cada sistema estudiado tiene un propio $w_{c}$, cuyos valores para el Pacífico oeste, el Pacífico este, el Océano Índico y el Atlántico son respectivamente 66,2; 63,7; 66,8 y 61,8 mm (Peters y Neelin, 2006a). Este último hecho es evidente en la Figura 3 pero se pierde con el reescalamiento de $w$, al multipicarlo por el factor $f_{w}^{i}$. El factor de reescalamiento $f_{P}^{i}$ reflejará la capacidad de cada región climática $i$ de generar precipitación.

LA GRANJA, Revista de ciencias de la vida, 15(1) 2012: 5-18.

(C) 2012, Universidad Politécnica Salesiana, Ecuador.

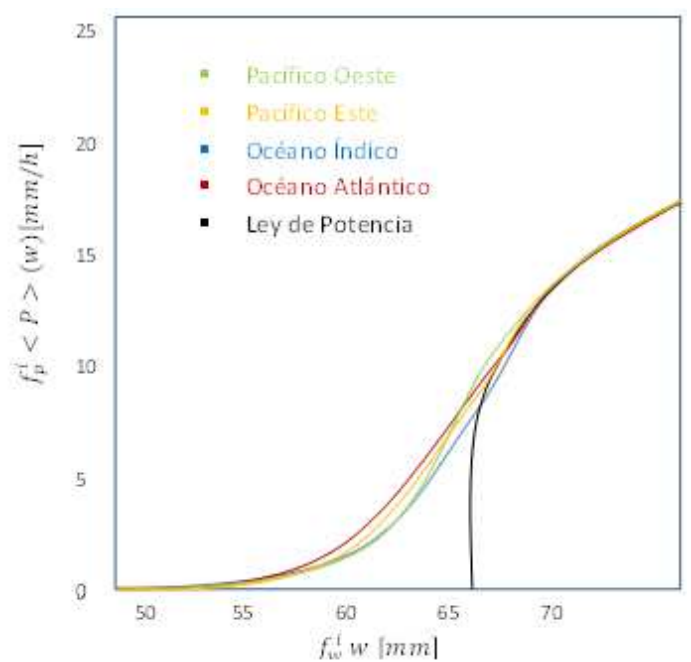

Figura 4. Parámetro de orden reescalado (promedio de la precipitación $\langle P\rangle(w)$ ) en función del parámetro de sintoninzación (vapor de agua $\langle w\rangle$ ), también reescalado para cada región climática estudiada en los trópicos. Es evidente que el comportamiento sigue una ley de potencia, marcada con la línea continua. Adaptado de Peters y Neelin (2006a).

Un trabajo posterior elaborado en el 2009 por Neelin et al. (2009) hace un estudio a profundidad de todas las variables importantes sobre estos sistemas climáticos; de este trabajo, es relevante destacar la no influencia de la temperatura sobre el comportamiento de ley de potencia, ya que se sigue encontrando un valor crítico y un exponente universal para diferentes temperaturas, tomadas a diferentes alturas de la tropósfera. Asimismo, se hace un estudio muy detallado de la dependencia del valor crítico del vapor de agua wc respecto a la temperatura, estudio que se muestra en la Figura 5.

Para visualizar el comportamiento de la ley de potencia, nos referimos a la Figura 6, donde se muestra la precipitación promedio en función del vapor de agua reducido: $\Delta w \equiv\left(w-w_{c}\right) / w_{c}$, para cada uno de los cuatro océanos estudiados, en un gráfico doblemente logarítmico. En este caso, es especialmente interesante que el exponente $b$ es universal, independiente de la región estudiada, e igual a 0,215 . Cabe resaltar que la ley de potencia se ajusta bastante bien, sin la necesidad de realizar un fine tunning, logrando virtualmente la misma pendiente para cada océano estudiado, independientemente de sus climatologías propias, con un error relativo de tan solo 0,02 . 


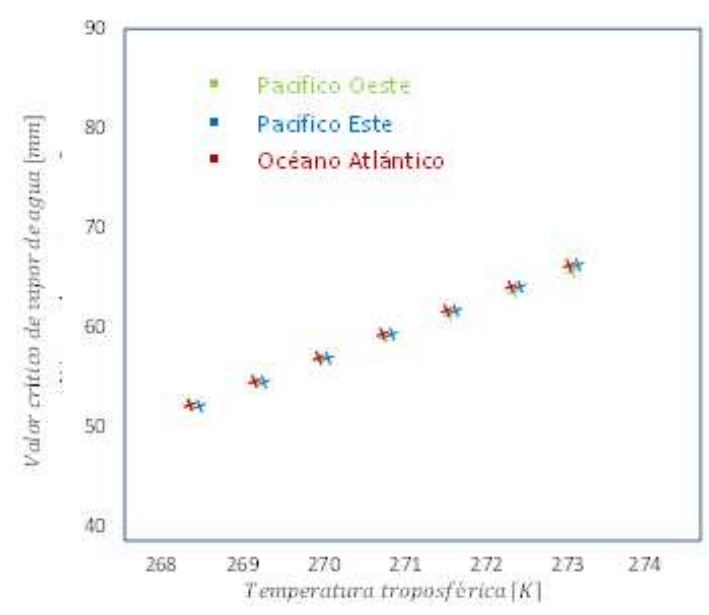

Figura 5. Columna de vapor de agua crítico wc en función de la temperatura troposférica, para cada uno de los océanos estudiados. Adaptado de Neelin et al. (2009).

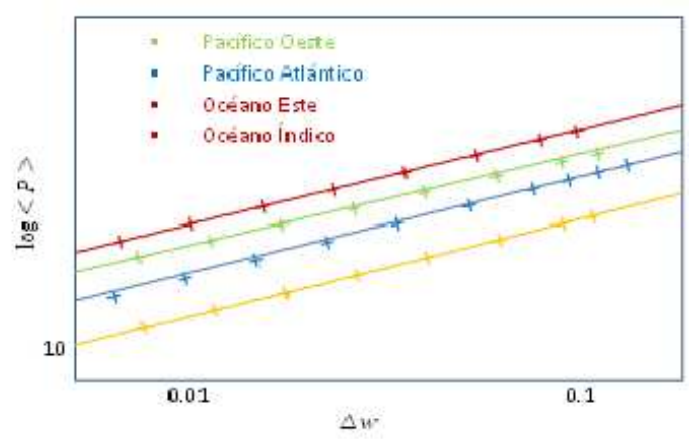

Figura 6. Escala doble logarítmica de la precipitación en función del vapor de agua reducido para los océanos $\mathrm{Pa}-$ cífico oeste (verde), este (rojo), Atlántico (azul) e Índico (rosado). Las líneas continuas son para guía, se tiene una pendiente de 0,215 , que se ajusta igualmente bien a cada región. Adaptado de Peters y Neelin (2006a).

\section{Criticalidad, complejidad adaptabilidad}

Si se quisiera definir a la complejidad, no se podrían aplicar este concepto a un sistema en donde todos los puntos son iguales a los demás (es decir no se puede aplicar a sistemas aleatorios, en donde no existe correlación entre los puntos). Zhang et al. (2011) ha aportado una útil concepción de un siste- ma complejo: aquel que tiene información en todas las escalas de longitud y tiempo, i.e. la complejidad es la información integrada $-\Sigma p \log p$ de un sistema sobre todos los posibles valores de tiempo o espacio. A través del formulismo de Zhang se ha determinado que un sistema es más complejo en el punto crítico. Es decir, Complejidad es Criticalidad. Y la SOC se puede interpretar como el hecho de que la naturaleza busca la máxima complejidad.

Así, ¿qué será entonces la adaptabilidad de un sistema complejo? Ya que en un sistema dinámico determinista no existen los conceptos de "propósito", "racionalidad", y por lo tanto "aprendizaje" y "adaptabilidad", la pregunta realmente debería ser cuáles son las características de los sistemas complejos que un observador puede interpretar como adaptabilidad.

Así, lo que se observa como "adaptabilidad o resilencia" de un sistema es su dinámica (avalanchas, terremotos, etc.) en función de los grados de libertad que lo forman como un sistema único, y que permiten que busque y se mantenga en un estado crítico (Bak y Sneppen, 1993).

\section{Criticalidad, entropía y negen- tropía}

Bajo otro contexto, es válido indicar que la entropía ha sido un concepto básico para el estudio del clima, como lo muestran los estudios de Peixoto et al. (1991) donde se muestra que la energía del sistema climático proveniente de la radiación solar iguala casi exactamente al flujo de radiación de onda larga que sale, mientras que los flujos de entropía que salen son 22 veces más grandes que los flujos de entropía asociados con la radiación entrante. De esta manera, se afirma que el sistema, cumpliendo la segunda ley de la termodinámica, genera entropía debido a la conducción de procesos irreversibles (Bleck et al., 2010; Benjamin et al., 2004).

Tan significativo como el concepto de la entropía es el de la negentropía, que ha nacido del desarrollo de la Teoría de la Información. En esta teoría se propone a la Entropía de Información de Shannon, la cual cuantifica el contenido de información transportada por un mensaje en términos de la mejor tasa de compresión sin pérdidas. Posteriormente, Brillouin en 1962, postula el principio de negentro-

LA GRANJA, Revista de ciencias de la vida, 15(1) 2012: 5-18.

(C) 2012, Universidad Politécnica Salesiana, Ecuador. 
pía en información a través de su libro Science and Information Theory, en el cual postula que adquirir o extraer información acerca de los microestados de un sistema, decrece la entropía, y como es necesario hacer trabajo para extraer la información, la entropía decrece localmente, pero aumenta en alguna otra parte, respetando así la Segunda Ley de la termodinámica (Roopun et al., 2008).

En fin, según Mahulikar y Herwing (2009), la negentropía termodinámica es el déficit específico de entropía de un sub-sistema ordenado dinámicamente, relativo con su entorno. Así, Jaynes (1957) ha validado la utilización del concepto de negentropía, no sólo en el estudio de sistemas climáticos sino en su pronóstico, como lo muestra el trabajo de Liu et al. (2011), en el que se reproduce con gran exactitud las trayectorias de los huracanes tropicales, como aquella trayectoria con mayor negentropía.

Estos conceptos desde sus fundamentos han estado ligados a los conceptos de auto-organización; fue Bernard en 1900, quien al calentar una capa fina de un fluido en reposo y someterlo a una diferencia de temperatura, con un flujo característico de calor, observó que pasado cierto valor crítico de diferencia de temperatura, el sistema llegaba a autoorganizarse abruptamente, formando patrones regulares de celdas convectivas de forma hexagonal. Sugirió entonces que todo sistema sometido a un flujo de entropía negativo (flujo negentrópico) está asociado a una auto-organización al pasar por un valor crítico. Tiempo después, en los años cuarenta, Schrödinger propuso en su monografía What is $\mathrm{Li}$ $f e$ ?, que la existencia de la vida dependía de un continuo aumento de la negentropía de los alrededores, y que este flujo era completamente significante para el sistema, ya que éste le permitía lograr su nivel de auto-organización

\section{Conclusiones y Recomendacio- nes}

Abordando los resultados desde el punto de vista meteorológico, se sugiere que las anteriores concepciones de $\mathrm{QE}$, base para la descripción dinámica y convectiva de la precipitación, y por lo tanto base de muchos programas computacionales de predicción numérica, deben extenderse e incluir teorías que involucran los conceptos de criticalidad autoorganizada y transiciones de fase, cuya utilidad ha sido abordada en esta revisión para el caso de la precipitación, sin olvidar la necesidad de complementar el estudio del sistema climático con otros conceptos termodinámicos desarrollados como es el caso de la entropía y la negentropía. Conceptos que han mostrado su gran capacidad descriptiva y predictiva en el sistema atmosférico.

Por otro lado, uno de los principales aportes de esta novel concepción radica en el modelamiento más adecuado y significativamente simple de la precipitación $\rangle P\langle(w)$, que través de la ecuación (8), lleva a determinar que la lluvia intensa es un fenómeno de naturaleza fractal.

Desde una comprensión más básica el hecho de que la precipitación intensa dependa únicamente de sobrepasar cierto umbral $w_{c}$, sin estar asociada directamente con un parámetro temporal, podría de alguna forma dificultar en un futuro la predicción de la ocurrencia de lluvias torrenciales, por ejemplo. Sin embargo, aún cabe determinar si el parámetro de sintonización $\mathrm{w}$ tiene alguna correspondencia más directa con escalas temporales que permitan predecir su evolución en el tiempo, y de esta manera generar de alguna forma sistemas de alerta a las precipitaciones intensas. Debido a que sí existe un monitoreo bastante completo del vapor de agua a través de sistemas satelitales a escala planetaria.

Por otro lado, no hay que olvidar que el comportamiento de la precipitación intensa respecto a la temperatura es un hecho conocido: la cantidad de vapor de agua en un volumen depende de la temperatura; de la termodinámica se desprende que mientras mayor sea la temperatura mayor cantidad de vapor de agua puede contener un sistema. Por esta razón, los investigadores en sus primeros trabajos, que van del 2004 al 2006: Bretherton et al. (2003); Peters y Neelin (2006a); Pruessner y Peters (2006), escogieron sistemas sobre océanos cuyas climatologías se caracterizan por tener las variaciones mínimas de temperatura.

Sin embargo, y para apoyar la robustez de la SOC, se muestra que la temperatura no influye en la transición de fase a la precipitación intensa siguiendo una ley de potencia, aún más se ha mostrado que el exponente logrado $b$ es universal. Y que lo que se ve afectado directamente por la temperatura es el valor crítico $w_{c}$, por lo tanto, se recomienda probar este marco teórico para sistemas con variabilidad térmica significativa, como los que se encuentran sobre tierra Condom et al. (2011). En estos casos 
será necesario precisar la dependencia lineal del valor de $w_{c}$ respecto a la temperatura, así como el parámetro a que determina la capacidad de cada sistema climático de lograr precipitaciones intensas.

En conclusión, esta recopilación plantea un novel formalismo para el estudio de los fenómenos complejos, cuyos resultados respecto a la SOC y la teoría de las transiciones de fase indican que nos encontramos más cerca del entendimiento de este fenómeno.

\section{Agradecimientos}

Los autores agradecen a Marco Bayas, Enrique Palacios, Marcos Villacís y Nicolás Vásquez por sus valiosos aportes en la discusión de los temas técnicos y teóricos de esta investigación.

\section{Referencias}

Adami, C. 1955. Self-organized criticality in living systems. Physical Letters A, 203(1): 29-32.

Arakawa, A. y W. Schubert. 1974. Interaction of a cumulus cloud ensemble with the large-scale environment, part I. . Journal Atmospheric Science, 31: 674-701.

Bak, P. 1996. How Nature Works: The science of self-organized criticality. Copernicus.

Bak, P. y K. Chen. 1991. Self-organized criticallity. Science Amercian, 246(1).

Bak, P. y K. Sneppen. 1993. Punctuated equilibrium and criticality in a simple model of evolution. Physical Review Letters, 71(24): 4083-4086.

Bak, P., C. Tang y K. Wiesenfeld. 1987. Selforganized criticality. Physical Review A, 38(1): 364-374.

Benjamin, Grell, Brown, Smirnova y R. Bleck. 2004. Mesoscale weather prediction with the RUC hybrid isentropic-terrain-following coordinate model. Mon. Weather Rev., 132: 473-494.

Bleck, Benjamin, Lee y MacDonald. 2010. On the use of an adaptive, hybrid-isentropic vertical coordinate in global atmospheric modeling. Mon. Weather Rev., 138: 2188-2210.
Bretherton, C., M. Peters y L. Back. 2003. Relationships between Water Vapor Path and Precipitation over the Tropical Oceans. Journal of Climate, 17.

Bretherton, C., M. Peters y L. E. Back. 2004. Relationships between water vapor path and precipitation over the tropical oceans. Journal Clim., 17.

Bunde, A., J. Eichner, R. Govindan, S. Halvin, E. Koscienly-Bunde, D. Rybski y D. Vjushin. 2003. Power-Law persistence in the Atmosphere Analysis and Applications. Nonextensive Entropy-Interdisciplinary Applications, New York Oxford University Press, 17.

Chistensen, K., H. J. Jensen y H. C. Fogedby. 1991. Dynamical and Spatial Aspects of Sandpile Celluar Automata. J. Stat. Phys., 63: 653-681.

Condom, T., P. Rua y J. C. Espinoza. 2011. Correction of TRMM 3B43 monthly precipitation data over the montainous areas of Peru during the period 1998-2007. The European Physical Journal B, 82: 83-89.

Cowan, G., D. Pines y D. Meltzer. 1994. Complexity: Methaphors, Models and Reality. SFI Studies in the Sciences of Complexity. Addison Wesley.

Grabowski. 2003. MJO-like coherent structures: Sensitivity simulations using the cloudresolving convection parameterization (CRCP). Journal of Atmospheric Sciences, 60: 847-864.

Ibañez, J. 2007. Física de Fractales, Geosfera, Edafosfera y Biosfera (La Criticalidad AutoOrganizada). $\mathrm{MI}+\mathrm{D}$.

Jaynes, E. 1957. Information theory and statistical mechanics. Phys. Rev., 105: 620-630.

Kagan, Y. 1992. Seismicity: Turbulence of solids. Nonlinear Sci. Today, 2: 1-13.

Kron, T. y T. Grund. 2009. Society as a Selforganized Critical System. Cybernetics and Human Knowing, 16: 65-82.

Liu, Y., C. Liu y D. Wang. 2011. Understanding Atmospheric Behaviour in Terms of Entropy: A Review of Applications of the Second Law of Thermodynamics to Meteorology. Entropy, 13: 211240 .

LA GRANJA, Revista de ciencias de la vida, 15(1) 2012: 5-18.

(C) 2012, Universidad Politécnica Salesiana, Ecuador. 
Lucas, C. 1999. Self-Organizing Systems. Usenet group comp.theory.self-org-sys.

Mandelbrot, B. 1982. The Fractal Geometry of Nature. W.H. Freeman, San Francisco.

Marro, J. y R. Dickman. 1999. Nonequilibrium Phase Transitions in Lattice Models. Cambridge Univiversity Press, Cambridge.

Neelin, D., O. Peters y K. Hales. 2009. The transition to strong convection. Journal of Atmospheric Science.

Neelin, D., O. Peters, J. Lin, K. Hales y C. Holloway. 2008. Rethinking convective quasi-equilibrium: observational constraints for stochastic convective schemes in climate models. Philosophical Transactions of the Royal Society A, 366: 25792602.

Newman, M. 2005. Power laws, Pareto distributions and Zipf's law. Contemporary Physics, $46(1)$.

Olami, Z., H. J. Feder y K. Christensen. 1992. Selforganized criticallity in a continuous, nonconservative cellular automaton modeling earthquakes. Phys. Rev. Lett., 68: 1464-1247.

Palacios, E. y S. Serrano. 2011. Validación de los Modelos de Cambio Climático hidrostáticos y no hidrostáticos sobre la climatología de Ecuador en las variables de precipitación y temperaturas extremas. La Granja, 13.

Palacios, E., S. Serrano y P. Núñez. 2009. Estudio de la climatología ecuatorial andina con métodos numéricos: pronósticos de tiempo, validaciones y recosntrucción de la atmósfera. La Granja, 10.

Paltridge, G. 1975. Global dynamics and climate: a system of minimum entropy exchange. Quart. J. Roy. Meteorol. Soc., 101: 475-484.

Parsons, D. B., K. Yoneyama y J. Redelsperger. 2000. The evolution of the tropical western Pacific ocean-atmosphere system following the arrival of a dry intrusion. Meteorological Society, 126: 517-548.

Peixoto, J., A. Oort, M. de Almeida y A. Tome. 1991. Entropy budget of atmosphere. Journal Geophys. Res., 96: 10981-10988.
Peters, O. y K. Christensen. 2002. Rain: Relaxations in the sky. Physical Review E.

Peters, O., C. Hertlein y K. Christensen. 2002. Complexity view of rainfall. Physical Review Letters, 88(1).

Peters, O. y D. Neelin. 2006a. Critical phenomena in atmospheric precipitation-Supplementary Information. nature physics.

Peters, O. y J. D. Neelin. 2006b. Critical phenomena in atmospheric precipitation. Nature physics, 2: 393-396.

Privman, V., P. Hohenberg y Aharony. 1991. A. in Phase Transitions and Critical Phenomena. Academic, New York, 14: 1-134.

Pruessner, G. y O. Peters. 2006. Self-organized criticality and absorbing states: Lessons from the Ising model. Physical Review E, 73(025106).

Puyeyo, S. 2011. Self organised criticality and the response of wildland fires to climate change. Climatic Change, 82: 131-161.

Ramirez, R. 2000. Autocriticalidad de los incendios forestales. Tesis Doctoral, Universidad Nacional de Estudios a Distancia.

Raup, M. D. 1986. Bilogical extinction in earth history. Science, 251: 1530-1532.

Reynoso, C. 2007. Complejidad y el Caos: Una exploración antropológica. Universidad de Buenos Aires.

Roopun, Kramer, Carracedo, Kaiser, Davies, Traub, Kopell y Whittington. 2008. Temporal interactions between cortical rhythms. Front. Neurosci., 2: 145-154.

Sekar, I. 2007. Realization of soc behavior in a dc glow discharge plasma. Physics Letters A, 360: 717-721.

Sethna, J. 2010. Entropy, order parameters and Complexity. Clarendon, Oxford.

Steward, I. 1997. Does God play dice? Penguin Books.

Turcotte, D. L. 1985. Collapse of loaded fractal trees. Nature, 313: 671. 
Wolf, B., D. Marks, E. Amital, D. Silberstein, B. Fisher, A. Tokay, J. Wang y L. Pippitt. 2005. Ground Validation for de Tropical Rainfall Measuring Mission (TRMM). American Meteorological Society.

Wolff, D. B., D. Marks, E. Amitai, D. Silberstein, B. Fisher, A. Tokay, J. Wang y J. Pippitt. 2004. Ground Validation for the Tropical Rainfall Measuring Mission (TRMM). American Meteorological Society.

Xu, K. y K. Emanuel. 1989. Is the tropical atmosphere conditionally unstable? . Monthly Weather Review, 117: 1471-1479.
Yeomans, J. 1992. Statistical Mechanics of Phase Transitions. Clarendon, Oxford.

Zhang, G., U. Tirnakli, L. Wang y T. Chen. 2011. Self organized criticality in a modified Olami-FederChristensen model. The European Physical Journal B, 82: 83-89.

Zhang, Y. y S. Klein. 2010. Mechanisms Affecting the Transition from Shallow to Deep Convection over Land: Inferences from Observations of the Diurnal Cycle Collected at the ARM Southern Great Plains Site. Journal of atmospheric sciences. 